\title{
BMJ Open Challenges in the acute identification of mild traumatic brain injuries: results from an emergency department surveillance study
}

\author{
Ilaria Pozzato (D) , ${ }^{1}$ Susanne Meares (D) , ${ }^{2}$ Annette Kifley, ${ }^{1}$ Ashley Craig (D) , ${ }^{1}$ \\ Mark Gillett, ${ }^{3}$ Kim Van Vu, ${ }^{1}$ Anthony Liang, ${ }^{1}$ Ian Cameron, ${ }^{1}$ Bamini Gopinath (1) ${ }^{1}$
}

To cite: Pozzato I, Meares S, Kifley A, et al. Challenges in the acute identification of mild traumatic brain injuries: results from an emergency department surveillance study. BMJ Open 2020;10:e034494. doi:10.1136/ bmjopen-2019-034494

- Prepublication history and additional material for this paper are available online. To view these files, please visit the journal online (http://dx.doi. org/10.1136/bmjopen-2019034494).

Received 24 September 2019 Revised 20 December 2019 Accepted 09 January 2020

D) Check for updates

C Author(s) (or their employer(s)) 2020. Re-use permitted under CC BY-NC. No commercial re-use. See rights and permissions. Published by BMJ.

${ }^{1}$ John Walsh Centre for Rehabilitation Research, Kolling Institute, University of Sydney, St Leonards, New South Wales, Australia

${ }^{2}$ Department of Psychology, Macquarie University, Sydney, New South Wales, Australia

${ }^{3}$ Emergency Department, Royal North Shore Hospital, Sydney, New South Wales, Australia

Correspondence to

Dr Bamini Gopinath;

bamini.gopinath@sydney.edu.au

\section{ABSTRACT}

Objectives To establish the proportion of mild traumatic brain injury (mTBI) diagnosis among people presenting to an emergency department (ED), to determine the accuracy of recorded ED diagnoses. We also aimed to describe challenges in mTBI case identification and its acute hospital management.

Design and setting A retrospective chart review of all ED attendances to a major trauma hospital, over a 9-month period (June 2015-February 2016).

Participants Adults aged 18-65 years consecutively presenting to an ED.

Primary outcome measures Proportion of mTBI diagnosis among ED attendances (ie, confirmed mTBI based on the WHO criteria or indeterminate $\mathrm{MTBI}$ based on secondary criteria), and proportion of accurately recorded mTBI diagnosis by ED clinicians (ie, 'mTBl', 'concussion'). Results Of $30479 \mathrm{ED}$ attendances, 351 (1.15\%) confirmed mTBI diagnosis and $180(0.6 \%)$ indeterminate diagnosis were identified. Only 81 (23.1\%) individuals with a confirmed mTBI had a 'mTBI diagnosis' clearly recorded in the medical notes. Of the allocated discharge diagnosis codes to the two identified cohorts, $89.8 \%$ were not indicative of $\mathrm{mTBI}$. Intracranial injuries were found in 31 (8.5\%) confirmed cases. Glasgow Coma Scale scores were consistently assessed in the ED but identified only 117 (33.3\%) confirmed mTBI cases. Post-traumatic amnesia (PTA) testing was able to confirm acute cognitive impairment in $113(62.1 \%)$ of those who were tested (182, $51.3 \%)$.

Conclusions mTBI is a common, but an under-recognised cause for ED attendance. Despite challenges, the use of an operational definition such as the WHO diagnostic criteria can improve accuracy in $\mathrm{mTBI}$ identification. Acute management may be enhanced by rapid assessment of PTA.

\section{INTRODUCTION}

Mild traumatic brain injuries (mTBI) are a serious public health problem that is referred to as a 'silent epidemic'. ' Though being the least severe of all brain injuries, identification is the most challenging, with mTBI often missed at diagnosis. ${ }^{2}$ Major barriers to mTBI identification are the wide variability in
Strengths and limitations of this study

- A systematic chart review of all emergency department attendances was employed to capture any possible mild traumatic brain injury (mTBI) case.

- The use of standard diagnostic criteria to establish the occurrence of mTBI diagnosis ensures accuracy in identification and comparability across existing research.

- This study provides novel data on proportions of rapid post-traumatic amnesia (PTA) screening in NSW, Australia, where there is written recommendation around PTA screening in all emergency departments.

- Collecting data from single hospital site limits generalisability of study findings.

- Given the retrospective design, conclusions on mTBI occurrence and accuracy of designation were limited by the availability of documented clinical information, with $\mathrm{mTBI}$ occurrence possibly being underestimated.

criteria used for diagnosis and the lack of sensitive standardised measures for identifying mTBI manifestations, which are commonly subtle and rapidly resolving. ${ }^{2}{ }^{3}$ Despite these limitations, the WHO best-evidence review estimated that hospital-treated mTBI are in the range of $100-300 / 100000$ population. ${ }^{4}$ Diagnosis and management of mTBI largely occur in an emergency department (ED). ${ }^{5}$ Little information exists, however, about the accuracy of mTBI identification in emergency settings. Two studies, conducted in three Canadian $\mathrm{EDs}^{6}$ and two EDs in the $\mathrm{USA}^{7}{ }^{7}$ found that up to $50 \%$ of patients sustaining mTBI received an inaccurate ED diagnosis. Poor identification likely impacts clinical management of these patients. Given trends in increasing ED attendances for head trauma ${ }^{89}$ there is a critical need for research that addresses the challenges in mTBI diagnosis. 
Another challenge for ED clinicians is the identification of mTBI cases at major risk of complications versus those who can be safely discharged..$^{10}$ Latest research suggests that these so-called minor injuries can have long-term impacts that extend beyond the anticipated 3-month time frame of cognitive recovery for uncomplicated cases, calling for urgent improvements in the acute management of mTBI. Long-term impacts include higher healthcare usage, ${ }^{11}$ psychosocial complications ${ }^{12} 13$ and in vulnerable subgroups chronic cognitive symptomatology ${ }^{1214}$ and neural cellular alterations ${ }^{15}{ }^{16}$ not easily detectable by routine radiological examinations that may increase the risk of neurodegeneration. ${ }^{17}$ EDs represent a crucial point where accurate identification and early management of these patients may prevent long-term personal and economic impacts.

Key steps to aid early and accurate identification and management of mTBI include enhanced consistency in diagnostic criteria and standardised assessment methods. ${ }^{3}$ An internationally recognised operational definition was developed by the WHO Task Force, ${ }^{3}{ }^{4}$ clearly outlining the four key clinical manifestations for mTBI diagnosis. These are: (i) level of consciousness, (ii) confusion or disorientation, (iii) post-traumatic amnesia (PTA) and (iv) transient neurological abnormalities, such as CT-detected intracranial injuries, the latter defined as complicated mTBI (about $10 \%$ of cases). ${ }^{18}$ Recommended objective measures to assess TBI 'severity' include conventional radiology to exclude structural lesions and the Glasgow Coma Scale (GCS) to monitor level of consciousness (ie, mTBI is defined as GCS scores of $13-15$ out of 15 and a loss of consciousness (LOC) of $\leq 30 \mathrm{~min}$ ). However, no clear guidance is given by the WHO on the clinical assessment of the other TBI diagnostic criteria. ${ }^{4}$ This particularly applies to PTA, which is recognised as the best prognostic indicator of mTBI outcomes. ${ }^{19} 20$

PTA is a complex clinical concept reflecting an acute transient cognitive dysfunction ${ }^{21}$ that presents not only as amnesia but more broadly as a period of inability to store new information, confusion, disorientation or behavioural changes. ${ }^{321}$ While standardised testing exists to assess the resolution of acute cognitive dysfunction (ie, PTA) these are rarely used in the acute management of mTBI patients because many protocols are too lengthy to be administered in ED settings. ${ }^{21}$ In NSW, Australia, the Abbreviated Westmead PTA Scale (A-WPTAS) ${ }^{21}$ was specifically designed for ED use and recommended statewide (NSW Ministry of Health, Initial management of closed head injury in adults, 2011) as a brief validated measure of PTA to improve identification of traumatic brain injury events among closed head injury patients with a GCS of 13-15 (NSW Ministry of Health, Initial management of closed head injury in adults, 2011). This measure includes five GCS orientation items plus a memory test of recall of three picture cards learnt on the first trial. The test is repeated hourly for up to 4 hours until optimal scores of 18 out of 18 are obtained (ie, 15 on the GCS, plus 3 on the memory test), indicating a resolution of
PTA, if present. Though the A-WPTAS has been shown to assist with a safer discharge of people with mTBI, by identifying cases with a GCS of $15 / 15$ who remain acutely cognitively impaired, ${ }^{20}$ and reducing hospitalisation and direct costs, ${ }^{22}$ its implementation to date appears inconsistent. Unpublished Australian data showed that rates of PTA screening in ED range from $0 \%$ to $31 \%,{ }^{10}$ while findings from a recent randomised controlled trial showed lower rates (ie, below 13\%). ${ }^{23}$ This highlights the need for further studies to investigate the extent and possible benefit of A-WPTAS implementation in emergency settings.

Given the current challenges in mTBI diagnosis and limitations of existing epidemiological research, this study primarily aimed to establish: (i) the occurrence of mTBI diagnosis among ED attendances (ie, meeting standard diagnostic criteria), and the proportion of these that received a clearly recorded $\mathrm{mTBI}$ diagnosis (ie, based on clinical notes and/or diagnosis codes). A secondary aim was to describe challenges in acute identification and management of mTBI such as the implementation of a validated measure for PTA screening in ED.

\section{METHODS}

This is a retrospective cohort study, employing chart review and standard WHO diagnostic criteria to define occurrence of mTBI among adults aged 18-65 years with ED attendances of a major trauma hospital in Sydney, Australia, over a 9-month period (from June 2015 to February 2016). Two independent chart auditors systematically screened all ED attendances within the study period and reviewed all recorded information in ambulance reports, ED and medical notes, to determine whether mTBI occurred. Details of the study method are available. ${ }^{24}$

The main outcomes were: (i) proportions of identified mTBI diagnosis, meeting WHO diagnostic criteria, among total ED attendances within 24 hours post-injury, meeting study age-range and time frame, and (ii) proportions of accurately recorded mTBI diagnoses by ED clinicians based on positive mTBI-related definition documented in the medical record. A confirmed mTBI diagnosis was ascertained based on the presence of any of the four mTBI manifestations (ie, level of consciousness, confusion/disorientation, PTA, transient neurological abnormalities), as expressed by the corresponding WHO criteria (table 1 and online supplementary table $1)^{421}$ : (i) a GCS of 13-15 30 min after injury or on later presentation to healthcare; and/or LOC of $\leq 30 \mathrm{~min}$; (ii) confusion/disorientation, (iii) $\mathrm{PTA}<24$ hours and/or (iv) CT-detected intracranial injuries not requiring neurosurgery, respectively.

Despite the uniqueness of this study in using a validated measure for PTA screening in ED, initial chart review indicated PTA testing was not consistently administered. Further, optimal scores obtained during ED stay would still not be able to identify cases whose PTA resolved 
Table 1 Clinical features of mTBI diagnosis (confirmed mTBI, $n=351$; indeterminate $m T B I, n=180$ ) who presented to ED, illustrated by WHO diagnostic criteria and secondary criteria

\begin{tabular}{|c|c|c|c|}
\hline & $\begin{array}{l}\text { Confirmed mTBI } \\
\text { (WHO criteria) } \\
\text { ( } n=351)\end{array}$ & $\begin{array}{l}\text { Indeterminate mTBI } \\
\text { (secondary criteria) } \\
\text { ( } \mathrm{n=180)}\end{array}$ & $\begin{array}{l}\text { Statistical } \\
\text { comparison }\end{array}$ \\
\hline & $\mathbf{N}(\%)$ & $\mathbf{N}(\%)$ & P value* \\
\hline mTBI WHO criteria & & & \\
\hline (i) Level of consciousness & & & \\
\hline Initial GCS at the scene/ED triage & & & \\
\hline 15 points & $232(66.1)$ & $180(100)$ & \\
\hline 14 points & $103(29.2)$ & - & \\
\hline 13 points & $14(4)$ & - & \\
\hline Missing & $2(0.57)$ & - & \\
\hline $\mathrm{LOC} \leq 30 \mathrm{~min}$ & & & \\
\hline No & $71(20.2)$ & - & \\
\hline Yes (witnessed/self-reported) & $185(52.7)$ & - & \\
\hline Missing & $50(14.2)$ & - & \\
\hline (ii) Confusion/disorientation & $97(27.6)$ & - & \\
\hline (iii) Amnesia <24 hours & & & \\
\hline No & $40(11.4)$ & - & \\
\hline Yes (observed/self-reported) & $229(65.2)$ & - & \\
\hline Missing & $78(22.2)$ & - & \\
\hline (iv) Intracranial injuries on brain CT & $31(8.8)$ & - & \\
\hline Multiple mTBI WHO criteria & $186(53)$ & - & \\
\hline mTBI secondary criteria & & & \\
\hline Queried LOC & $45(12.8)$ & $12(6.7)$ & $<0.05$ \\
\hline Queried amnesia & $4(1.1)$ & $3(1.7)$ & 0.564 \\
\hline PTA testing w/ optimal scores & $32(9.1)$ & $45(25)$ & $<0.001$ \\
\hline Post-concussion symptoms $\dagger$ & $183(52.1)$ & $133(73.9)$ & $<0.001$ \\
\hline Headache & $149(42.4)$ & $128(71.1)$ & \\
\hline Nausea/vomiting & $103(29.3)$ & $79(43.9)$ & \\
\hline Dizziness & $44(12.5)$ & $58(32.2)$ & \\
\hline Fatigue & $2(0.6)$ & $1(0.6)$ & \\
\hline Memory problems & $5(1.4)$ & $1(0.6)$ & \\
\hline Concentration problems & $4(1.1)$ & $2(1.1)$ & \\
\hline Other & $81(23.1)$ & 78 (43.3) & \\
\hline Transient neurological abnormalities $†$ & $28(8)$ & $22(12.2)$ & \\
\hline Multiple mTBI secondary criteria & - & $32(17.8)$ & \\
\hline
\end{tabular}

The data contain occasional missing data values, which are assumed to be random.

${ }^{*} \chi 2$, z-test, t-test.

†Proportion of valid cases.

ED, emergency department; GCS, Glasgow Coma Scale; LOC, loss of consciousness; mTBI, mild traumatic brain injury; PTA, post-traumatic amnesia.

early post-injury ${ }^{2}$ (ie, optimal scores meaning that PTA, if present, had resolved). These cases could, instead, be identified by any evidence of PTA manifestations (ie, any gap in memory, period of confusion/disorientation, behavioural changes) documented by ambulance and emergency staff in their clinical observations. Therefore, to ascertain the presence of PTA as criterion for mTBI occurrence, a summary PTA-related mTBI designation (online supplementary table 2) was developed by crosschecking any PTA-related neurological and behavioural disturbances documented in medical records, from the time of injury to hospital discharge. A positive PTA 
designation was defined by any of the following source: acute cognitive impairment on A-WPTAS testing, observed behavioural change suggestive of PTA (eg, repetitive questioning, combative behaviour), as well as any observed/ self-reported gap in memory, or confusion/disorientation thereby fulfilling two of the WHO criteria. ${ }^{321}$

In the absence of any documented WHO criterion, indeterminate evidence of $\mathrm{mTBI}^{25}$ was defined based on the presence of any secondary criteria ${ }^{20}$ : (i) optimal scores (ie, 18/18) on the second trial of the A-WPTAS indicating that PTA, if present, had resolved, (ii) symptoms that may correspond to 'post-concussion symptoms' but which are not specific to mTBI, ${ }^{24}$ (iii) transient neurological abnormalities (excluding intracranial injuries not requiring surgery), which are not common findings or clinical features of $\mathrm{mTBI}^{23}$ and are not recommended as standalone mTBI criteria, ${ }^{2}$ or (iv) queried LOC/amnesia.

The accuracy of the diagnosis given by ED clinicians to the identified individuals with mTBI diagnosis was assessed by the presence of any recorded 'mTBI', 'concussion', 'post-concussion symptoms/syndrome' diagnoses in medical notes. ${ }^{26}$ In addition, allocation of relevant mTBI-related discharge diagnosis codes (SNOWMED codes) was also explored, to inform how much routinely collected administrative data could be useful for brain injury diagnostic purpose.

\section{Patient and public involvement}

Patients or the public were not involved in the design, or conduct, or reporting, or dissemination of our research.
RESULTS

\section{Identified cases with mTBI diagnosis}

During the study period, 30479 adults aged 18-65 years attended the ED and were screened (figure 1). Of the 587 mTBI-related ED presentations initially identified, 56 cases were excluded due to: $(n=27)$ self-discharge or unclear evidence of mTBI, $(n=8)$ confounding factors (eg, intubation, psychosis, medical comorbidities) or possible moderate TBI (eg, LOC/amnesia of unclear duration). Also excluded were 21 (3.9\%) individuals who represented for the same mTBI event. Among total ED attendances, $351(1.15 \%)$ confirmed mTBI diagnoses and an additional $180(0.6 \%)$ cases with insufficient/ indeterminate mTBI evidence were identified (online supplementary figure 1$)$. Of these, two people $(0.4 \%)$ sustained multiple mTBI (ie, repetitive mTBI). Sociodemographic, injury-related and acute management details are illustrated in tables 1-3.

A clearly recorded mTBI diagnosis in ED records ${ }^{26}$ was present only in $23.1 \%(\mathrm{n}=81)$ of confirmed mTBI and $18.9 \%(\mathrm{n}=34)$ of indeterminate cases. Similarly, among the 551 ED diagnosis codes (ie, SNOWMED codes) (table 4) allocated to the two identified cohorts, the most commonly used code descriptor was 'injury of head' $(n=145,26.3 \%)$. Only 56 of these codes $(10.2 \%)$ were specifically indicative of brain injury occurrence, with 'concussion' being the most common ( $\mathrm{n}=26,46.4 \%)$. The remaining codes mostly reflected intracranial injury findings (see also online supplementary table 3, which shows the full list of ED discharge diagnosis codes).
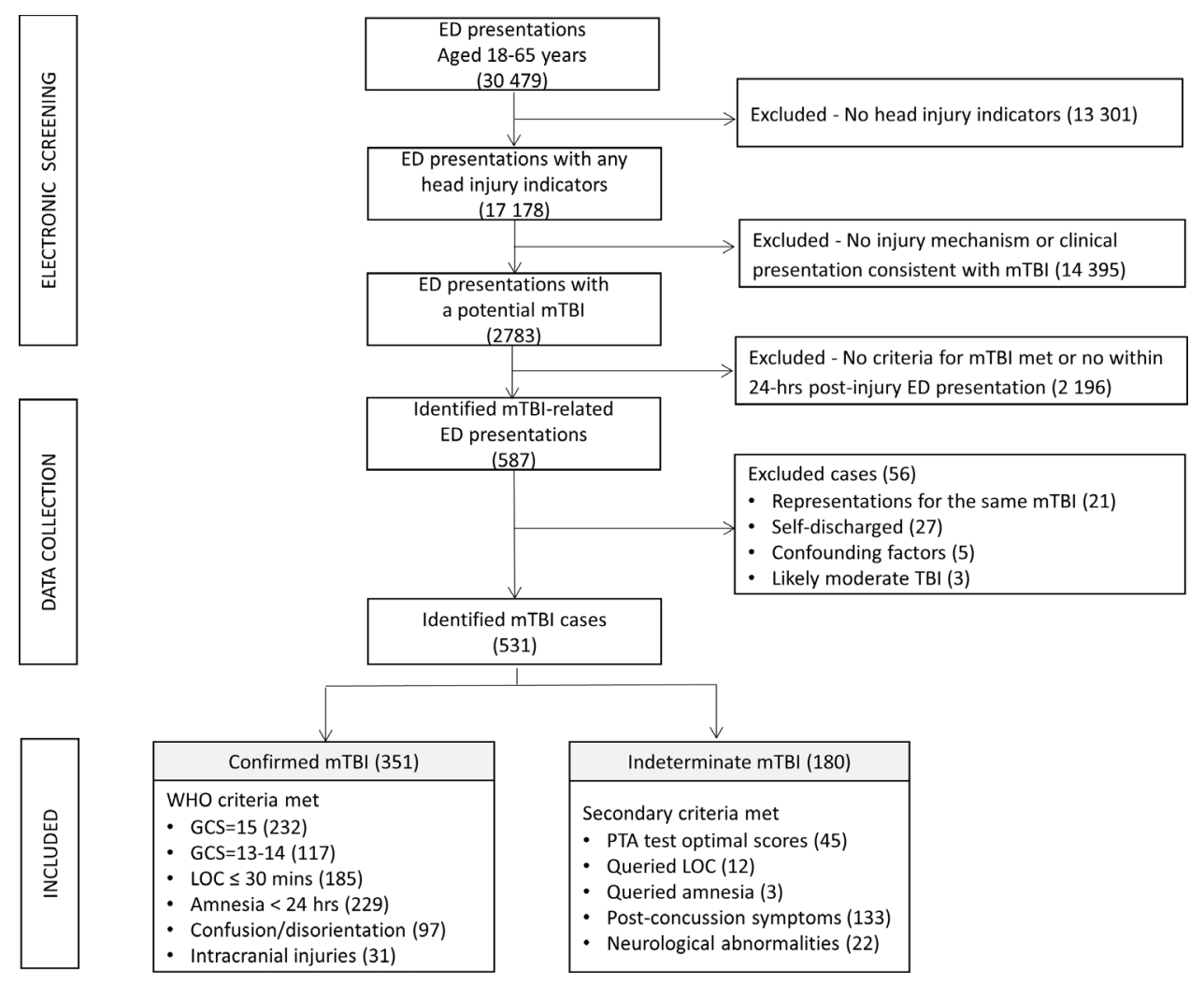

Figure 1 Study recruitment flow chart. 
Table 2 Sociodemographic and injury-related information of identified cases with a confirmed mTBI diagnosis ( $n=351)$ and indeterminate mTBI diagnosis $(n=180)$ who presented to ED

\begin{tabular}{|c|c|c|c|}
\hline & $\begin{array}{l}\text { Confirmed mTBI } \\
\text { (WHO criteria) } \\
\text { ( } \mathrm{n}=351 \text { ) }\end{array}$ & $\begin{array}{l}\text { Indeterminate mTBI } \\
\text { (secondary criteria) } \\
\text { ( } n=180)\end{array}$ & Statistical comparison \\
\hline & $\mathbf{N}(\%)$ & $\mathrm{N}(\%)$ & P value* \\
\hline \multicolumn{4}{|l|}{ Sociodemographics } \\
\hline $\begin{array}{l}\text { Age (years), mean (SD) } \\
\text { (median, IQR) }\end{array}$ & $\begin{array}{l}39.9(14.2) \\
(40.8,26.5-52)\end{array}$ & $\begin{array}{l}36.1(13.1) \\
(34.7,24.1-44.5)\end{array}$ & $<0.01$ \\
\hline \multicolumn{4}{|l|}{ Age groups (years) } \\
\hline $18-24$ & $71(20.2)$ & $52(28.9)$ & 0.02 \\
\hline $25-29$ & $38(10.8)$ & $15(8.3)$ & 0.36 \\
\hline $30-34$ & $36(10.3)$ & $26(14.4)$ & 0.16 \\
\hline $35-39$ & $24(6.8)$ & $19(10.6)$ & 0.13 \\
\hline $40-44$ & $44(12.5)$ & $24(13.3)$ & 0.79 \\
\hline $45-49$ & $34(9.7)$ & $12(6.7)$ & 0.24 \\
\hline $50-54$ & $44(12.5)$ & $9(5)$ & $<0.01$ \\
\hline $55-59$ & $25(7.1)$ & $11(6.1)$ & 0.66 \\
\hline $60-65$ & $35(10)$ & $12(6.7)$ & 0.21 \\
\hline Sex & & & $<0.001$ \\
\hline Male & $254(72.4)$ & $90(50)$ & \\
\hline Female & $97(27.6)$ & $90(50)$ & \\
\hline Country of birth & & & 0.50 \\
\hline Australia & $230(65.5)$ & $113(62.8)$ & \\
\hline Other & $118(33.6)$ & $66(36.7)$ & \\
\hline Language spoken at home & & & 0.11 \\
\hline English & 334 (95.2) & $165(91.7)$ & \\
\hline Other & $17(4.8)$ & $15(8.3)$ & \\
\hline Marital status & & & 0.11 \\
\hline Married/defacto & $171(48.7)$ & $83(46.1)$ & \\
\hline Other & $180(51.3)$ & $97(53.9)$ & \\
\hline Mental health history $\dagger$ & $61(17.4)$ & $23(12.8)$ & 0.17 \\
\hline Substance abuse history† & $36(10.2)$ & $1(0.5)$ & \\
\hline \multicolumn{4}{|l|}{ Injury-related details } \\
\hline Injury mechanism & & & 0.35 \\
\hline Fall & $137(39)$ & $56(31.1)$ & \\
\hline Assault & $27(7.7)$ & $14(7.8)$ & \\
\hline Work & $9(2.6)$ & $7(3.9)$ & \\
\hline Sport & $52(14.8)$ & $25(13.9)$ & \\
\hline Other & $27(7.7)$ & $34(18.9)$ & \\
\hline Motor vehicle crash & 99 (28.2) & $44(24.4)$ & $<0.01$ \\
\hline Driver & $28(28.3)$ & $15(34.1)$ & \\
\hline Passenger & $6(6.1)$ & $6(13.6)$ & \\
\hline Motorbike rider & $8(8.1)$ & $10(22.7)$ & \\
\hline Bicyclist & $44(44.4)$ & $5(11.4)$ & \\
\hline Pedestrian & $10(10.1)$ & $5(11.4)$ & \\
\hline Other & $3(3)$ & $3(6.8)$ & \\
\hline Reported impact to the head & & & $<0.001$ \\
\hline
\end{tabular}




\begin{tabular}{|c|c|c|c|}
\hline & $\begin{array}{l}\text { Confirmed mTBI } \\
\text { (WHO criteria) } \\
(\mathrm{n}=351)\end{array}$ & $\begin{array}{l}\text { Indeterminate mTBI } \\
\text { (secondary criteria) } \\
(n=180)\end{array}$ & Statistical comparison \\
\hline & $\mathbf{N}(\%)$ & N (\%) & P value* \\
\hline No & $2(0.6)$ & $1(0.5)$ & \\
\hline Yes & $269(76.6)$ & $164(91.1)$ & \\
\hline Soft tissue laceration & $165(47)$ & $55(30.6)$ & $<0.001$ \\
\hline Fracture & $87(24.8)$ & $24(13.3)$ & $<0.01$ \\
\hline Ligamentous & $2(0.6)$ & $2(1.1)$ & 0.49 \\
\hline Dislocation & $3(0.8)$ & 0 & 0.21 \\
\hline
\end{tabular}

The data contain occasional missing data values that are assumed to be random.

${ }^{*} \chi 2$, z-test, t-test.

†Proportion of valid cases.

$\mathrm{ED}$, emergency department; mTBI, mild traumatic brain injury.

Confirmed mTBI cases that were given a clearly recorded $\mathrm{mTBI}$ diagnosis and/or a discharge code suggestive of mTBI were more likely to (online supplementary table 4$)$ be a non-traffic-crash related mTBI $(\mathrm{p}<0.05)$, be admitted to a ward $(p<0.05)$, have CT-detected intracranial injuries $(p<0.0001)$, present with headaches $(p<0.05)$ and/or concentration problems $(\mathrm{p}<0.05)$, and be recommended for follow-up care $(\mathrm{p}<0.01)$. Furthermore, those with a clearly recorded mTBI diagnosis were more likely to have been tested for PTA ( $\mathrm{p}=0.0003)$, while those with an allocated discharge code suggestive of mTBI were more likely to have a clearly written mTBI diagnosis in their ED records as well $(\mathrm{p}=0.04)$.

\section{Injury-related characteristics}

Cases with a confirmed mTBI diagnosis met the following WHO criteria: initial GCS of 13-14 (ie, at the scene 30-min post-injury/at ED admission; $\mathrm{n}=117$; 33.3\%), LOC (ie, witnessed/self-reported; $\mathrm{n}=185 ; 52.7 \%$ ), amnesia (ie, observed/self-reported; $\mathrm{n}=229 ; 65.2 \%$ ), confusion/ disorientation $(\mathrm{n}=97 ; 27.6 \%)$ and CT-detected intracranial injuries $(\mathrm{n}=31 ; 8.8 \%)$. Multiple WHO criteria were present in 186 cases (53\%) (table 1). Cases with an indeterminate mTBI diagnosis met the following secondary criteria in the absence of WHO criteria: optimal scores of $18 / 18$ on the A-WPTAS $(n=45 ; 25 \%)$, presence of postconcussion symptoms $(133 ; 73.9 \%)$, transient neurological abnormalities $(n=22 ; 12.2 \%)$ queried LOC $(n=12$; $6.7 \%)$ and/or queried amnesia $(n=3 ; 1.7 \%)$. Multiple secondary criteria were present in 32 cases $(17.8 \%)$.

Fall was the most common cause of mTBI in both confirmed $(39.1 \%)$ and indeterminate $(31.1 \%)$ groups, followed by motor vehicle crash (28.2\%-confirmed; 24.4\%-indeterminate). Alcohol or drug use in association with the injury was self-reported or clinically observed in $127(36.2 \%)$ confirmed cases compared with only 19 $(10.6 \%)$ of indeterminate cases $(\mathrm{p}<0.001)$.

\section{Acute hospital management details}

Brain imaging was undertaken in $75.8 \%$ of cases with a confirmed mTBI diagnosis and $40.6 \%$ of cases with an indeterminate mTBI $(\mathrm{p}<0.001$; table 3$)$. Only 182 $(51.8 \%)$ individuals with a confirmed mTBI were tested for PTA (ie, A-WPTAS or WPTAS). Of these, the majority $(106 ; 58.4 \%)$ had a PTA duration of $>1$ to 12 hours, 32 (17.8\%) obtained optimal scores of 18/18 (ie, did not fail A-WPTAS testing) and $37(20.4 \%)$ had an unknown designation due to incomplete/missing documentation. Median time to the first PTA testing was 3.7 (2.36.1) hours post-injury for confirmed mTBI diagnosis and 2.5 (1.7-4.9) hours for indeterminate mTBI.

The summary PTA-related mTBI designation (online supplementary table 2) including any documented positive PTA-related findings (ie, neurological and behavioural disturbances) in the medical records identified a total of $260(74.1 \%)$ confirmed mTBI cases with PTA. The majority $(89.8 \%)$ were identified based on two WHO criteria of observed/self-reported amnesia (ie, any gap in memory) and/or period of confusion/disorientation, with a further nine people deemed in PTA only due to failing the A-WPTAS $(n=8)$ or due to reported behavioural changes in medical records, $(n=1)$ (ie, repetitive questioning). All these nine cases, except one, also met at least one of the other mTBI WHO criteria (eg, LOC, GCS=13-14, intracranial injuries). 
Table 3 Acute hospital management details of identified cases with a confirmed mTBI diagnosis $(n=351)$ and indeterminate mTBI diagnosis $(n=180)$ who presented to ED

\begin{tabular}{|c|c|c|c|}
\hline & $\begin{array}{l}\text { Confirmed mTBI } \\
\text { (WHO criteria) } \\
\text { ( } \mathrm{n}=351 \text { ) }\end{array}$ & $\begin{array}{l}\text { Indeterminate mTBI } \\
\text { (secondary criteria) } \\
\text { ( } n=180)\end{array}$ & $\begin{array}{l}\text { Statistical } \\
\text { comparison }\end{array}$ \\
\hline & $\mathrm{N}(\%)$ & $\mathrm{N}(\%)$ & P value* \\
\hline ED management details & & & \\
\hline ED arrival mode & & & $<0.001$ \\
\hline By ambulance & $250(71.2)$ & $56(31.1)$ & \\
\hline Other & $101(28.8)$ & $124(68.9)$ & \\
\hline Triage category & & & $<0.001$ \\
\hline 1. Seen immediately & $24(6.8)$ & $4(2.2)$ & \\
\hline 2. Within $10 \mathrm{~min}$ & $170(48.4)$ & $42(23.3)$ & \\
\hline 3. Within $30 \mathrm{~min}$ & $116(33.1)$ & $64(35.6)$ & \\
\hline 4. Within 1 hour & $40(11.4)$ & $69(38.3)$ & \\
\hline 5. Within 2 hours & $1(0.3)$ & $1(0.6)$ & \\
\hline Intubation† & $1(0.3)$ & 0 & 0.47 \\
\hline ICU admissiont & $7(2)$ & $1(0.6)$ & 0.19 \\
\hline Length of ED stay (hours), median (IQR) & $5.8(4-8.6)$ & $3.8(2.6-5.7)$ & $<0.001$ \\
\hline Length of hospital stay (days), median (IQR) & $3.4(1.9-6.5)$ & $2(0.9-7.9)$ & \\
\hline Discharge destination & & & $<0.001$ \\
\hline Discharged home & $133(37.9)$ & $127(70.6)$ & \\
\hline Admitted to ED & $121(34.5)$ & $36(20)$ & \\
\hline Admitted to ward & $97(27.6)$ & $17(9.4)$ & \\
\hline Location of initial GCS $†$ & & & $<0.001$ \\
\hline At the scene 30-min post-injury & $216(61.5)$ & $52(28.9)$ & \\
\hline At ED presentation & $130(37)$ & $127(70.6)$ & \\
\hline Brain CT performed $\dagger$ & $266(75.8)$ & $73(40.6)$ & $<0.001$ \\
\hline PTA measured $\dagger$ & $182(51.9)$ & $46(25.6)$ & $<0.001$ \\
\hline A-WPTAS & $169(92.8)$ & $44(95.6)$ & \\
\hline WPTAS & $19(10.4)$ & $2(4.4)$ & \\
\hline Location of PTA testing & $(n=182)$ & $(n=46)$ & \\
\hline In ED & $166(91.2)$ & $44(95.6)$ & 0.364 \\
\hline In ward & $28(15.4)$ & $2(4.4)$ & $<0.05$ \\
\hline Time to PTA testing (hours), median (IQR) & $3.7(2.3-6.1)$ & $2.5(1.7-4.9)$ & $<0.05$ \\
\hline PTA classification based on PTA testing & $(n=182)$ & $(n=46)$ & $<0.001$ \\
\hline Optimal scores/did not fail & $32(17.8)$ & $40(87)$ & \\
\hline 6-30 min & $2(1.1)$ & - & \\
\hline $31-60 \mathrm{~min}$ & $1(0.1)$ & - & \\
\hline$>1-12$ hours & $106(58.4)$ & - & \\
\hline$>12-24$ hours & $4(2.2)$ & - & \\
\hline Unknown/incomplete/missing & $37(20.4)$ & $6(13)$ & \\
\hline Head injury advice given $\dagger$ & $166(47.3)$ & $120(66.7)$ & $<0.001$ \\
\hline Follow-up recommendations & $128(36.5)$ & $43(23.9)$ & $<0.01$ \\
\hline Representations to ED (within 1 month) & $13(3.7)$ & $8(4.4)$ & \\
\hline Recorded mTBI diagnosis in the ED records & & & 0.47 \\
\hline No & $269(76.9)$ & $146(81.1)$ & \\
\hline Yes & $81(23.1)$ & 34 (18.9) & \\
\hline
\end{tabular}




\begin{tabular}{lll}
$\begin{array}{l}\text { Confirmed mTBI } \\
\text { (WHO criteria) } \\
(n=351)\end{array}$ & $\begin{array}{l}\text { Indeterminate mTBI } \\
(\text { secondary criteria) } \\
(n=180)\end{array}$ & $\begin{array}{l}\text { Statistical } \\
\text { comparison }\end{array}$ \\
\cline { 2 - 3 }$(\%)$ & $N(\%)$ & Pvalue $^{*}$ \\
\hline
\end{tabular}

The data contain occasional missing data values that are assumed to be random.

${ }^{*} \chi 2$, z-test, t-test.

†Proportion of valid cases.

A-WPTAS, Abbreviated Westmead Post-Traumatic Amnesia Scale; ED, emergency department; GCS, Glasgow Coma Scale; ICU, intensive care unit; mTBI, mild traumatic brain injury; PTA, post-traumatic amnesia; WPTAS, Westmead Post-Traumatic Amnesia Scale.

In both groups, people tested for PTA were more likely to (online supplementary table 5) be transported to ED by ambulance $(p<0.01)$; sustain a traffic-related mTBI $(p<0.001)$; be admitted to ED/ward $(p<0.0001)$. Confirmed mTBI cases were more likely be tested for PTA in the presence of other mTBI signs such as LOC $(\mathrm{p}=0.005)$, amnesia $(\mathrm{p}<0.0001)$ and brain imaging $(\mathrm{p}=0.003)$ with positive findings $(\mathrm{p}<0.0001)$.

\section{DISCUSSION}

By using the WHO operational criteria, our study reports an occurrence of confirmed mTBI diagnosis among ED total attendances of $1.2 \%$ (351/30 479). These findings correspond to the $1.1 \%-1.3 \%$ proportion observed in a preliminary study, which used the same criteria and methods for TBI diagnosis ${ }^{2024}$ therefore confirming the robustness of the proposed WHO surveillance system for acute mTBI identification. A similar proportion of mTBI cases seeking emergency care $(1.9 \%$; 670/35 096) was also reported in a prospective cohort study conducted in a large metropolitan ED in New York. ${ }^{27}$ This study employed the alike 1993 American College of Rehabilitation Medicine criteria, ${ }^{27}$ as operational definition, suggesting that using standard diagnostic criteria can enhance consistency in mTBI identification and comparability of study findings.

Worryingly, only $23.1 \%(81 / 351)$ of our identified cohort with a confirmed mTBI diagnosis (ie, meeting the WHO criteria) had an accurate mTBI diagnosis documented in the medical records (ie, written diagnosis of 'concussion', 'mTBI'). The proportion of accurate diagnoses was much lower than reported in two previous prospective studies conducted in Canada $^{6}$ and the USA, ${ }^{7}$ respectively, being $\geq 50 \%$. While using a retrospective design could account for these differences, global challenges certainly exist in the acute identication of 'minor' TBI events. This study contributes by providing unique Australian data and suggests that adopting standard criteria and the assessment of PTA provide so far the best approach to improve accuracy of mTBI diagnosis.

Poor accuracy in mTBI identification in $\mathrm{ED}^{317}$ could affect current estimates of 100-300/100 000 reported in a WHO review, ${ }^{4}$ hence underestimating the 'true' incidence of hospital-treated mTBI. Surveillance systems, such as accurate administrative databases, are recommended strategies to tackle this problem. However, the use of discharge diagnosis codes, such as ICD coding, in hospital databases has previously been shown not to be sensitive in detecting mTBI. ${ }^{26}{ }^{27}$ Our results confirm this gap. Only $10.7 \%$ (59/551) of ED discharge diagnosis codes (SNOWMED codes) allocated to the identified cohorts with either a confirmed or indeterminate mTBI diagnosis were indicative of mTBI. Despite limitations in the number of diagnoses able to be recorded (ie, maximum two SNOWMED codes), there seems to be a trend for ED clinicians to better identify the more 'severe' injuries, that is, those showing positive CT findings, being admitted to a ward and receiving follow-up care recommendations. By contrast, uncomplicated mTBI appears to be overlooked, by not receiving an accurate designation in ED records or accurate coding. There was also an interchangeable use of terms like 'concussion', '(mild/minor) head injury' and 'mTBI', as also shown in previous studies ${ }^{317} 27$ that suggest a poor clarity in the distinction between those having a traumatic brain injury versus simple head injuries, thus reiterating the scarce utility of administrative data in mTBI identification.

While the WHO criteria can be regarded as a reliable system for the identification of individuals who sustained a mTBI, there were challenges in its application. ${ }^{2}$ First, it was unclear how to interpret LOC or amnesia when it was not witnessed/observed as per WHO recommendations for mTBI identification. It is likely that injured people report a LOC or amnesia interchangeably ${ }^{2}$; thus, a self-reported LOC/amnesia at the time of the injury suggests a confirmed mTBI diagnosis. Conversely, when LOC/amnesia was queried by a physician this more likely suggests indeterminate evidence of mTBI.

PTA is the most important TBI prognostic indicator, yet the most challenging to evaluate because it encompasses a series of acute cognitive impairment signs and symptoms. This study is unique in its way of screening for PTA in ED by means of a validated measure. However, standard PTA testing was only available in about half of confirmed mTBI cases $(51.9 \%)$, though this was considerably higher than previously reported PTA screening rates (up to $31 \%$ )..$^{10} 23$ Also, optimal scores of $18 / 18$ on the A-WTPAS were obtained in $17.8 \%$ of those who were tested. While optimal scores clearly indicate the absence of acute cognitive dysfunction at the time of assessment (ie, a person is not in PTA), these 
Table 4 Top 25 ED diagnosis codes (SNOWMED codes) and ED diagnosis codes indicative of $\mathrm{mTBI}$, for the overall $\mathrm{mTBI}$ cohort (confirmed $\mathrm{mTBI}$ cases, $\mathrm{n}=351$; indeterminate $\mathrm{mTBI}, \mathrm{n}=180$ )

\begin{tabular}{|c|c|c|}
\hline Top 25 ED diagnosis codes description & $\mathbf{N}$ & $\%$ \\
\hline Injury of head (disorder) & 145 & 26.3 \\
\hline Motor vehicle accident victim (finding) & 49 & 8.9 \\
\hline Minor head injury (disorder) & 39 & 7.1 \\
\hline Traumatic injury (disorder) & 37 & 6.7 \\
\hline Falls (finding) & 35 & 6.4 \\
\hline Concussion (disorder) & 26 & 4.7 \\
\hline Headache (finding) & 11 & 2.0 \\
\hline Facial laceration (disorder) & 10 & 1.8 \\
\hline Victim of physical assault (finding) & 9 & 1.6 \\
\hline Alcohol intoxication (disorder) & 8 & 1.5 \\
\hline Falling injury (finding) & 8 & 1.5 \\
\hline Fractured nasal bones (disorder) & 8 & 1.5 \\
\hline Laceration of head (disorder) & 8 & 1.5 \\
\hline Post-concussion syndrome (disorder) & 7 & 1.3 \\
\hline Injury of face (disorder) & 6 & 1.1 \\
\hline Subarachnoid haemorrhage (disorder) & 6 & 1.1 \\
\hline Neck pain (finding) & 5 & 0.9 \\
\hline Backache & 4 & 0.7 \\
\hline Intracranial injury without skull fracture (disorder) & 4 & 0.7 \\
\hline Laceration of forehead (disorder) & 4 & 0.7 \\
\hline Closed fracture of clavicle (disorder) & 3 & 0.5 \\
\hline Dizziness (finding) & 3 & 0.5 \\
\hline Fracture of maxilla (disorder) & 3 & 0.5 \\
\hline Fracture of rib (disorder) & 3 & 0.5 \\
\hline Injury of neck (disorder) & 3 & 0.5 \\
\hline \multicolumn{3}{|l|}{ ED diagnosis codes indicative of mTBI } \\
\hline Concussion (disorder) & 26 & 4.7 \\
\hline Post-concussion syndrome (disorder) & 7 & 1.3 \\
\hline Subarachnoid haemorrhage (disorder) & 6 & 1.1 \\
\hline Intracranial injury without skull fracture (disorder) & 4 & 0.7 \\
\hline Subdural haematoma (disorder) & 3 & 0.5 \\
\hline Brief loss of consciousness (finding) & 2 & 0.4 \\
\hline Cerebral haemorrhage (disorder) & 1 & 0.2 \\
\hline Contusion of cerebrum (disorder) & 1 & 0.2 \\
\hline $\begin{array}{l}\text { Crushing injury of skull and intracranial contents } \\
\text { (disorder) }\end{array}$ & 1 & 0.2 \\
\hline Epidural haemorrhage (disorder) & 1 & 0.2 \\
\hline Intracranial haemorrhage (disorder) & 1 & 0.2 \\
\hline Loss of consciousness (finding) & 1 & 0.2 \\
\hline Transient global amnesia (finding) & 1 & 0.2 \\
\hline Traumatic subdural haemorrhage (disorder) & 1 & 0.2 \\
\hline
\end{tabular}

ED, emergency department; mTBI, mild traumatic brain injury.

cannot exclude that PTA was present before that time, not being informative for mTBI diagnostic purpose.

Therefore, this study used a summary PTA-related mTBI designation, accounting for the positive presence of any
PTA-related neurological and behavioural disturbances recorded in medical records. ${ }^{31}$ Using this indicator, we found 260 of the 351 cases with a confirmed mTBI diagnosis were deemed to be in PTA. Of these, the majority $(89.8 \%)$ was based on the presence of observed/selfreported amnesia (ie, a gap in memory) or confusion/ disorientation (ie, meeting two of the four WHO criteria), while nine $(2.6 \%)$ cases were further identified based only on evidence of acute cognitive impairment (ie, failing PTA testing; $n=8)$ or observed behavioural changes $(n=1)$, the latter being repetitive questioning that is typically an indirect sign of PTA. These additional cases, except one, all met at least one of the other WHO criteria (eg, LOC, GCS=1314 , intracranial injuries) for mTBI diagnosis.

Overall, these findings reiterate that the WHO criteria together constitute the most reliable surveillance system for mTBI identification and provide useful information to specifically identify cases whose PTA may have resolved by the time of ED admission. In addition, the implementation of PTA testing, providing objective estimates of acute cognitive impairment, may assist in monitoring recovery progress towards a safer discharge and enhance diagnostic accuracy of cases where mTBI indicators are unclear or unavailable. The administration of brief PTA testing (ie, the A-WTPAS) as an extension of the GCS, which is usually assessed at the scene by the ambulance staff, ${ }^{21}$ could provide a more accurate estimate of the presence and duration of PTA, thus of mTBI occurrence.

Another challenge was the assessment of transient neurological abnormalities, other than intracranial injuries. Ruff et al suggested these abnormalities in isolation do not constitute a strong basis for mTBI diagnosis because they are not common or typical features of mTBI. ${ }^{2}$ Thus, these were considered as indeterminate evidence of mTBI. The WHO also recommends excluding cases whose TBI manifestations can be affected due to other factors. ${ }^{28}$ Unlike other confounders (eg, intubation, psychiatric disorder), the influence of alcohol/drug on mTBI manifestations was particularly difficult to assess due to the lack of objective blood level measurements. Overall, these cases accounted for $36.2 \%$ of confirmed mTBI, that is, in the range of previous findings $(30 \%-$ $60 \%) .{ }^{29}{ }^{30}$ These findings confirm intoxication is a major confounding affecting accurate identification of mTBI in busy ED settings, with day of injury blood alcohol level being associated with failure on PTA assessment, ${ }^{20}$ a longer duration of LOC and decreased GCS scores. ${ }^{31}$ Differentiation of mTBI in these individuals in the ED setting is likely to be facilitated by the potential implementation of blood-based biomarkers. ${ }^{32}$

This study confirms that issues exist in identifying the mildest TBIs, ${ }^{2}$ whose clinical manifestations may resolve within $<15 \mathrm{~min}$ post-injury according to the American Academy of Neurology classification. ${ }^{2}$ Considering the amount of missing or non-informative/optimal indicators among cases with a confirmed mTBI in this study, as also reported by previous research, ${ }^{33}$ along with PTA measured 2-3 hours post-injury, ${ }^{21}$ it is likely that rapid-resolving 
LOC/amnesia were missed with a bias towards more severe mTBI. Secondary criteria were established to identify cases with indeterminate evidence of TBI. These cases constituted $0.6 \%$ of ED total attendances and, interestingly, $18.9 \%$ of these received a positive mTBI diagnosis by ED clinicians. Another study using a similar (probabilistic) approach found delayed functional recovery in the group with debatable mTBI compared with healthy and trauma controls, ${ }^{25}$ raising concerns around the need for identifying and treating less-severe mTBI that may appear to not meet diagnostic criteria.

Intracranial injuries were found in $31(8.8 \%)$ cases with a confirmed mTBI diagnosis. ${ }^{18}$ Brain CT was performed in $75.8 \%$, plus in $40 \%$ of cases with an indeterminate mTBI. ${ }^{18}$ Clinical assessment remains the gold standard for mTBI identification, with PTA testing being the most promising measure. Among those who were tested, the A-WPTAS was able to detect acute cognitive impairment in $62.1 \%$ of cases $(113 / 182)$, while the GCS was able to detect only $33.5 \%$ of cases $(117 / 349) .{ }^{20}$ This study further suggests that when PTA is measured it increases the likelihood of an accurate mTBI designation provided by ED clinicians. Implementation of PTA testing in ED settings should be, thus, extended to all individuals with a possible $\mathrm{mTBI}^{21}$ to reduce the risk of missed opportunity for mTBI identification and to contribute to more accurate clinical decision making and safer discharge of patients.

\section{Study limitations}

Major strengths of this study were the use of standard diagnostic criteria for the identification of $\mathrm{mTBI}$ and the systematic screening of any cases with a possible mTBI diagnosis among ED attendances. However, the retrospective design is limiting as we might not have captured important information on confounding factors or mTBI indicators. Similarly, the absence of documented information in medical records does not necessarily imply that standard diagnostic criteria or assessment protocols were not applied by ED clinicians. Generalisability of findings is limited by the following selection bias ${ }^{30}$ : a working-age population, 9-month audit period and using only a single hospital site. Some of these issues will be addressed by conducting a multi-site study in the future.

\section{CONCLUSIONS}

The findings from this study indicate that mTBI is likely to be under-diagnosed in an emergency care setting. This study confirms the use of an operational definition, such as the WHO operational criteria as a reliable surveillance system for acute identification of mTBI, although challenges still exist in its meticulous application. Identification, prognosis and acute management of individuals with mTBI would be greatly enhanced by the implementation of standardised PTA screening (eg, A-WPTAS) early after injury. Improvements in clinical and administrative designation of these injuries require the use of these data to monitor and address long-term health and economic impacts of mTBI.

Contributors All authors-IP, SM, AK, AC, MG, KVV, AL, IC, BG—provided inputs in study design. IP, KVV, AL, AK, SM and BG were involved in data collection and data analysis. IP and BG were responsible for publication writing. All authors reviewed and approved the final version of this manuscript.

Funding This study has received research grant support from the Ramsay Research and Teaching Fund—Northern Sydney Local Health District, Sydney, Australia. The funding source had no role in study design, data collection and analysis, decision to publish and in the writing of this manuscript.

Competing interests None declared.

Patient consent for publication Not required.

Ethics approval Ethical approval was obtained from the Northern Sydney Local Health District Ethics, Sydney, Australia (LNR/16/HAWKE/388; LNRSSA/16/ HAWKE/389).

Provenance and peer review Not commissioned; externally peer reviewed.

Data availability statement All data relevant to the study are included in the article or uploaded as supplementary information.

Open access This is an open access article distributed in accordance with the Creative Commons Attribution Non Commercial (CC BY-NC 4.0) license, which permits others to distribute, remix, adapt, build upon this work non-commercially, and license their derivative works on different terms, provided the original work is properly cited, appropriate credit is given, any changes made indicated, and the use is non-commercial. See: http://creativecommons.org/licenses/by-nc/4.0/.

\section{ORCID iDs}

Ilaria Pozzato http://orcid.org/0000-0002-8752-1460

Susanne Meares http://orcid.org/0000-0002-3859-9974

Ashley Craig http://orcid.org/0000-0001-7647-7604

Bamini Gopinath http://orcid.org/0000-0003-3573-359X

\section{REFERENCES}

1 Goldstein M. Traumatic brain injury: a silent epidemic. Ann Neurol 1990;27:327.

2 Ruff RM, Iverson GL, Barth JT, et al. Recommendations for diagnosing a mild traumatic brain injury: a national Academy of neuropsychology education paper. Arch Clin Neuropsychol 2009;24:3-10.

3 Kristman VL, Borg J, Godbolt AK, et al. Methodological issues and research recommendations for prognosis after mild traumatic brain injury: results of the International collaboration on mild traumatic brain injury prognosis. Arch Phys Med Rehabil 2014;95:S265-77.

4 Cassidy JD, Carroll L, Peloso P, et al. Incidence, risk factors and prevention of mild traumatic brain injury: results of the who collaborating centre Task force on mild traumatic brain injury. $J$ Rehabil Med 2004;36:28-60.

5 Michelson EA, Huff JS, Loparo M, et al. Emergency department time course for mild traumatic brain injury workup. West J Emerg Med 2018;19:635-40.

6 Rowe BH, Eliyahu L, Lowes J, et al. Concussion diagnoses among adults presenting to three Canadian emergency departments: missed opportunities. Am J Emerg Med 2018;36:2144-51.

7 Powell JM, Ferraro JV, Dikmen SS, et al. Accuracy of mild traumatic brain injury diagnosis. Arch Phys Med Rehabil 2008;89:1550-5.

8 Marin JR, Weaver MD, Yealy DM, et al. Trends in visits for traumatic brain injury to emergency departments in the United States. JAMA 2014;311:1917-9.

9 Gaw CE, Zonfrillo MR. Emergency department visits for head trauma in the United States. BMC Emerg Med 2016;16:5.

10 Bosch M, McKenzie JE, Mortimer D, et al. Implementing evidencebased recommended practices for the management of patients with mild traumatic brain injuries in Australian emergency care departments: study protocol for a cluster randomised controlled trial. Trials 2014;15:1-20.10.1186/1745-6215-15-281

11 Kirsch NL, de Leon MB, Maio RF, et al. Characteristics of a mild head injury subgroup with extreme, persisting distress on the Rivermead Postconcussion symptoms questionnaire. Arch Phys Med Rehabil 2010;91:35-42.

12 Theadom A, Starkey N, Barker-Collo S, et al. Population-Based cohort study of the impacts of mild traumatic brain injury in adults four years post-injury. PLoS One 2018;13:e0191655. 
13 Stein MB, Jain S, Giacino JT, et al. Risk of posttraumatic stress disorder and major depression in civilian patients after mild traumatic brain injury: a TRACK-TBI study. JAMA Psychiatry 2019;76:249-58.

14 Karr JE, Areshenkoff CN, Garcia-Barrera MA. The neuropsychological outcomes of concussion: a systematic review of meta-analyses on the cognitive sequelae of mild traumatic brain injury. Neuropsychology 2014;28:321-36.

15 Arneson D, Zhang G, Ying Z, et al. Single cell molecular alterations reveal target cells and pathways of concussive brain injury. Nat Commun 2018:9:3894.

16 Hocke LM, Duszynski CC, Debert CT, et al. Reduced functional connectivity in adults with persistent Post-Concussion symptoms: a functional near-infrared spectroscopy study. $J$ Neurotrauma 2018;35:1224-32.

17 Gardner RC, Yaffe K. Epidemiology of mild traumatic brain injury and neurodegenerative disease. Mol Cell Neurosci 2015;66:75-80.

18 Stiell IG, Clement CM, Grimshaw JM, et al. A prospective clusterrandomized trial to implement the Canadian $\mathrm{CT}$ head rule in emergency departments. CMAJ 2010;182:1527-32.

19 Tellier A, Marshall SC, Wilson KG, et al. The heterogeneity of mild traumatic brain injury: where do we stand? Brain Inj 2009;23:879-87.

20 Meares S, Shores EA, Smyth T, et al. Identifying posttraumatic amnesia in individuals with a Glasgow coma scale of 15 after mild traumatic brain injury. Arch Phys Med Rehabil 2015;96:956-9.

21 Meares S, Shores EA, Taylor AJ, et al. Validation of the abbreviated Westmead post-traumatic amnesia scale: a brief measure to identify acute cognitive impairment in mild traumatic brain injury. Brain Inj 2011;25:1198-205.

22 Watson CE, Clous EA, Jaeger M, et al. Introduction of the abbreviated Westmead post-traumatic amnesia scale and impact on length of stay. Scand J Surg 2017;106:356-60.

23 Bosch M, McKenzie JE, Ponsford JL, et al. Evaluation of a targeted, theory-informed implementation intervention designed to increase uptake of emergency management recommendations regarding adult patients with mild traumatic brain injury: results of the net cluster randomised trial. Implement Sci 2019;14:4.

24 Pozzato I, Cameron ID, Meares S, et al. A surveillance study to determine the accuracy of mild traumatic brain injury diagnosis in an emergency department: protocol for a retrospective cohort study. BMJ Open 2017;7:e016222.

25 Korley FK, Diaz-Arrastia R, Falk HJ, et al. Prevalence of incomplete functional and symptomatic recovery among patients with head injury but brain injury debatable. J Neurotrauma 2017;34:1531-8.

26 Voss JD, Connolly J, Schwab KA, et al. Update on the epidemiology of concussion/mild traumatic brain injury. Curr Pain Headache Rep 2015;19:32

27 Bazarian JJ, Veazie P, Mookerjee S, et al. Accuracy of mild traumatic brain injury case ascertainment using ICD-9 codes. Acad Emerg Med 2006;13:31-8.

28 Cassidy JD, Boyle E, Carroll LJ. Population-Based, inception cohort study of the incidence, course, and prognosis of mild traumatic brain injury after motor vehicle collisions. Arch Phys Med Rehabil 2014;95:S278-85.

29 Brennan JH, Bernard S, Cameron PA, et al. Ethanol and isolated traumatic brain injury. J Clin Neurosci 2015;22:1375-81.

30 Luoto TM, Tenovuo O, Kataja A, et al. Who gets recruited in mild traumatic brain injury research? J Neurotrauma 2013;30:11-16.

31 Yue JK, Ngwenya LB, Upadhyayula PS, et al. Emergency department blood alcohol level associates with injury factors and six-month outcome after uncomplicated mild traumatic brain injury. J Clin Neurosci 2017;45:293-8

32 Yue JK, Yuh EL, Korley FK, et al. Association between plasma GFAP concentrations and MRI abnormalities in patients with CT-negative traumatic brain injury in the TRACK-TBI cohort: a prospective multicentre study. Lancet Neurol 2019;18:953-61.

33 Sharpe S, Kool B, Shepherd M, et al. Mild traumatic brain injury: improving quality of care in the paediatric emergency department setting. J Paediatr Child Health 2012;48:170-6. 